\title{
Predicting Success of Two-Stage Exchange for Prosthetic Joint Infection Using C-Reactive Protein/Albumin Ratio
}

\author{
Cierra S. Hong $\mathbb{D}^{1},{ }^{1}$ Sean P. Ryan $\mathbb{D}^{1},{ }^{1}$ Jonathan A. Gabor, ${ }^{2}$ Michael A. Bergen, \\ Ran Schwarzkopf $\left(1,{ }^{2}\right.$ and Thorsten M. Seyler ${ }^{1}$ \\ ${ }^{1}$ Duke University Medical Center, Durham, NC, USA \\ ${ }^{2}$ NYU Langone Health, New York City, NY, USA \\ Correspondence should be addressed to Cierra S. Hong; cierra.hong@duke.edu
}

Received 18 October 2018; Revised 1 March 2019; Accepted 18 March 2019; Published 2 May 2019

Academic Editor: Benjamin Blondel

Copyright (C) 2019 Cierra S. Hong et al. This is an open access article distributed under the Creative Commons Attribution License, which permits unrestricted use, distribution, and reproduction in any medium, provided the original work is properly cited.

Two-stage exchange is most commonly used for treatment of prosthetic joint infections (PJI) but, this may fail to eradicate infections. C-reactive protein/albumin ratio (CAR) has been used to predict survival and operative success in other surgical subspecialties and so, we assess the association between CAR and reimplantation success during two-stage revision for PJI defined by the Musculoskeletal Infection Society following a primary total hip (THA) or knee (TKA) arthroplasty. From January, 2005 to December, 2015, two institutional databases were queried and patient demographics, antibiotic duration, C-reactive protein, and albumin were collected prior to reimplantation. Two-stage revisions were considered successful if patients were off of antibiotics and did not require a repeat surgery. CAR was available for 79 patients (34 hips and 46 knees) with 61 successful two-stage revisions and 18 failures. The average CAR for patients with successful reimplantation was $1.2(0.2,3.0)$ compared to $1.0(0.4,3.2)$ for treatment failure. However, this was not statistically significant $(\mathrm{p}=0.766)$. Therefore, CAR is not applicable in predicting the prognosis of two-stage revisions for PJI in total arthroplasty but other preoperative inflammatory-based prognostic scores should be explored.

\section{Introduction}

In the United States, total hip arthroplasty (THA) and total knee arthroplasty (TKA) are two of the most commonly performed surgeries that treat arthritic pain and improve a patient's functional status. The number of procedures is projected to grow to about half a million and 1.5 million by 2020 , respectively [1]. However, this also increases the risk for postoperative complications, including prosthetic joint infections (PJIs), an uncommon but expensive and debilitating complication. From 2001 to 2009, the incidence of PJIs annually in THA and TKA increased from about $2 \%$ to $2.18 \%$, almost a twofold increase in the number of infected cases, which led to an additional $\$ 200$ million in inpatient care costs in the United States [2].

There are multiple strategies to treat and minimize PJI and its associated morbidity and mortality. They include the following medical and surgical managements: debridement, antibiotic, and implant retention (DAIR); one-stage arthroplasty exchange (resection prosthesis followed by reimplantation at the time of explant); two-stage arthroplasty exchange (resection prosthesis followed by reimplantation at a later time point from the explant); and resection arthroplasty $[3,4]$. Arthrodesis, amputation, and/or antimicrobial suppression are reserved for chronic or resistant infections [57]. In the United States, two-stage exchange is the preferred procedure for eradicating PJIs due to the strong literature support which shows successful outcomes in THA [8] and TKA $[9,10]$. However, despite at least a $70 \%$ infection-free survival over 10 years $[9,11]$, those who are not successfully treated with a two-stage revision arthroplasty experience severe complications that can result in soft tissue deficiencies, arthrodesis, amputations, or death [12]. This led to studies investigating risk factors, such as polymicrobial infections and multiple revision surgeries prior to explant of the primary arthroplasty, that predict two-stage revision failures in THA 
TABLE 1: Demographic and laboratory data of successful and failed two-stage revision cohorts.

\begin{tabular}{lccc}
\hline & Successful Two-Stage Revision & Failed Two-Stage Revision & $\mathrm{N}=18$ \\
& $\mathrm{~N}=61$ & $61.0(54.0,66.0)$ & $\mathrm{p}$-value \\
\hline Age (years at primary surgery) & $61.0(56.0,68.0)$ & $11(61.1)$ & 0.578 \\
Gender (female) & $28(45.9)$ & $4.3(1.8,10.0)$ & 0.257 \\
C-reactive Protein (mg/L) & $4.4(0.8,9.1)$ & $3.8(3.5,4.2)$ & 0.713 \\
Albumin (g/dL) & $3.7(3.1,4.0)$ & $1.0(0.4,3.2)$ & 0.263 \\
CRP/Albumin Ratio (CAR) & $1.2(0.2,3.0)$ & & 0.766 \\
\hline
\end{tabular}

and TKA in order to optimize surgical and medical management of these patients $[13,14]$.

In other surgical specialties, novel preoperative systemicinflammation based prognostic scores evaluating C-reactive protein (CRP) and albumin have been shown to predict surgical and overall survival outcomes in addition to risk for disease recurrence after oncologic resections [15-17]. Specifically, the CRP albumin ratio (CAR) has been shown to successfully predict prognosis in many types of cancers most likely because it combines a hallmark of tumorigenesis, inflammation, and preoperative nutritional status [18-21]. However, to our knowledge, CAR has not been investigated in assessing two-stage reimplantation outcomes for PJI in total arthroplasty. In this study, we aim to [1] report the preoperative CAR from patients undergoing a two-stage reimplantation for PJI from two different institutions and [2] assess the correlation with successful postoperative outcomes. We hypothesized that patients who had a successful treatment of their PJI after a two-stage revision for THA or TKA will have elevated preoperative CAR values.

\section{Materials and Methods}

From January 2005 to December 2015, two institutional databases at tertiary referral centers were retrospectively queried for patients who had a two-stage exchange for PJI after a primary THA or TKA. Patients were included for analysis only if they (1) met either one of the two major or four of the six minor criteria for PJI as defined by the Musculoskeletal Infection Society (MSIS) [22]; (2) completed an explant of their primary THA or TKA and reimplantation at a different surgery date; and (3) had a serum CRP and serum albumin value that were collected within one month of each other and were completed no later than one year before their explant. A total of 79 patients met these inclusion criteria and were retrospectively enrolled in the study. One patient had a two-stage revision for both a primary THA and TKA.

In addition to laboratory data including serum CRP and albumin, clinical demographics, such as age and gender, and antibiotic management were collected. A two-stage exchange arthroplasty was classified successful based off an international multidisciplinary consensus defining successful PJI eradication published by Diaz-Ledezma et al. known as the Delphi consensus criteria [23]. The consensus included the following: (1) no additional surgery to the affected joint after reimplantation; (2) complete PJI eradication which includes no life-long antibiotics for antimicrobial suppression or reinfection by the same organism; and (3) no causes of death related to PJI (i.e., sepsis).

Statistical analysis was performed using Wizard Pro for Mac (E. Miller, Chicago, IL). A univariable logistic regression analysis was performed to assess the primary endpoint, which was whether the two-stage exchange was successfully treating PJI at the two institutions included in the study. Continuous data are presented as median (lower quartile; upper quartile) and were analyzed with a Mann Whitney test between the two cohorts (successful versus unsuccessful twostage revision) while categorical data are presented as count (percent) and were analyzed with chi-squared test. The results were considered statistically significant when $\mathrm{p}<0.05$.

\section{Results}

From two institutional databases, a total of 79 patients (34 primary THA; 46 primary TKA) developed a PJI as defined by the MSIS and completed a two-stage exchange from 2005 to 2015. Sixty-one of these revisions successfully treated the patient's PJI whereas 18 total joint arthroplasty (5 THA; 13 TKA) required additional surgeries and/or chronic antimicrobial suppression (Figure 1). Demographic data, such as age and gender, in addition to laboratory values of interest are listed in Table 1 for these patients.

Between the two cohorts treated with two-stage exchange for PJI, the median CRP $(p=0.713)$ and albumin $(p=0.263)$ were not significantly different. Additionally, the preoperative CAR was not statistically significant between the cohort with a successful two-stage reimplantation compared to those with a failed reimplantation (successful CAR $1.2(0.2,3.0)$ versus failed CAR $1.0(0.4,3.2) ; \mathrm{p}=0.766)$. In the univariable logistic regression analysis, when considered in isolation, neither CRP (OR 1.00, 95\% CI 0.97-1.02; $\mathrm{p}=0.671$ ) nor albumin (OR $0.99,95 \%$ CI $0.09-1.05 ; \mathrm{p}=0.946$ ) adequately predicted failure of revision. Similarly, CAR did not predict failure (OR 0.975, 95\% CI 0.90-1.06; $\mathrm{p}=0.561$ ).

Forty-two patients (53.2\%) had CAR obtained within 30 days of surgery. Thirty of these patients had successful reimplantation and 12 had two-stage exchange revision failures with a CAR median of $1.20(0.19,3.43)$ and 1.03 $(0.45,2.38)$, respectively. There was no significant difference in preoperative CAR between these two groups $(\mathrm{p}=0.945)$ (Table 2).

\section{Discussion}

PJI, also known as periprosthetic joint infection, can compromise the prosthesis and surrounding bone and soft tissue 
TABLE 2: CAR of successful and failed two-stage revision cohorts within 30-day postoperative period.

\begin{tabular}{lccc}
\hline & Successful Two-Stage Revision & Failed Two-Stage Revision & $\mathrm{N}=12$ \\
\hline CRP/Albumin Ratio (CAR) & $\mathrm{N}=30$ & $1.03(0.45,2.38)$ & 0.945 \\
\hline
\end{tabular}
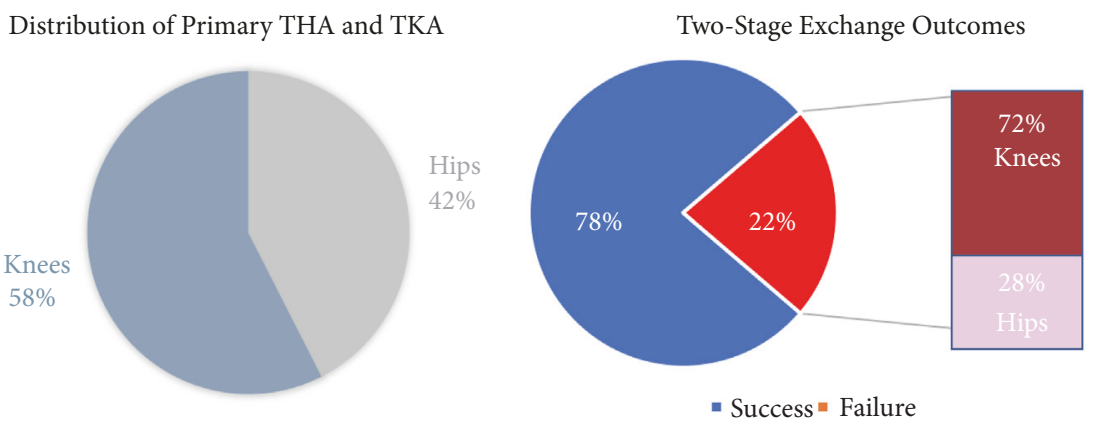

FIGURE 1: Left: distribution of patients included in study cohort. Right, distribution of two-stage exchange outcomes after primary THA and TKA.

structures, which significantly increases a patient's morbidity and can lead to patient mortality $[4,24]$. In 2011, the MSIS created the major and minor criteria in order to standardize the definition of PJI. It consists of periprosthetic cultures, elevated lab values including C-reactive protein (CRP) and erythrocyte sedimentation rate (ESR), and the presence of a sinus tract into the affected joint [22]. As previously mentioned, in the United States, in addition to antibiotic management, PJI is commonly managed with two-stage exchange arthroplasty due to its success with infection eradication and postoperative survival. However, the outcomes are variable with a reported failure rate up to a third of two-stage knee reimplantations [25] and a tenth of two-stage hip reimplantations [3]. Risk factors including the type of organism causing the PJI [26, 27] and preoperative synovial fluid characteristics [28] have been investigated and shown to predict two-stage exchange failures (Table 3). Another factor that has been studied is the timing of reimplantation after the first-stage explant is completed. Currently, the results remain inconclusive due to the lack of consistency between past studies in regard to the definition of PJI diagnosis and two-stage exchange failure and perioperative surgical and antibiotic treatment protocols between different institutions. For example, Sabry et al. [12] showed that, in patients with primary TKA PJIs, a longer time between explant and reimplantation was associated with infection recurrence. The reported median duration between the two stages associated with the latter was 103 days (range 2-470 days) whereas Rezaie et al. [29] showed the opposite result. Their analysis determined that the time between the two stages in THA and TKA PJI did not have an impact in predicting two-stage exchange failure.

The literature demonstrates multiple risk factors that lead to two-stage revision failures for THA and TKA including obesity, diabetes mellitus, and immunosuppression [39]. However, not only are these risk factors common for different surgical procedures $[40,41]$ but they also provide no prognostic value for two-stage revisions for PJI. On the other hand, many oncologic surgical specialties have shown that the novel preoperative scores involving systemic-inflammatory markers are valuable for prognosis $[42,43]$. This is likely due to the stimulation of inflammatory and acute-phase proteins in addition to the chemokines, cytokines, and immune cells, all of which contribute to tumor progression and dissemination [44]. In a similar manner, PJI stimulates the inflammatory response and systemic markers and can cause many of the classic clinical symptoms associated with this process such as fevers, erythematous warm joints, and pain [4].

Systemic-inflammatory markers were initially investigated individually as prognostic factors as seen with septic patients. Sepsis, which develops from a local infection that becomes systemic, is an inflammatory process that causes acute organ dysfunction and involves many of the factors mentioned above such as acute-phase proteins, chemokines and cytokines [45]. Simple prognostic factors including interleukin-6 (IL-6) [32] and D-dimer (Table 4) were shown to predict mortality in these patients. Eventually, this led to the development of novel systemic prognostic scores, which encompass a multifactorial presentation of the patient, to predict sepsis mortality $[35,38]$. The preoperative systemic-inflammatory based prognosis score, CAR, which predicts 180-day mortality in patients with sepsis [38], was of particular interest because it has successfully predicted prognosis in patients undergoing surgery for soft tissue sarcomas [18], esophageal [19], colorectal [20], and pancreatic cancers [21] and has outperformed other scores including neutrophil/lymphocyte ratio, platelet/lymphocyte ratio, and modified Glasgow Prognostic Score in gastric cancer [46]. Individually, elevated CRP [47] and hypoalbuminemia [39] have been associated with failed two-stage exchange outcomes but the present study showed that the preoperative 


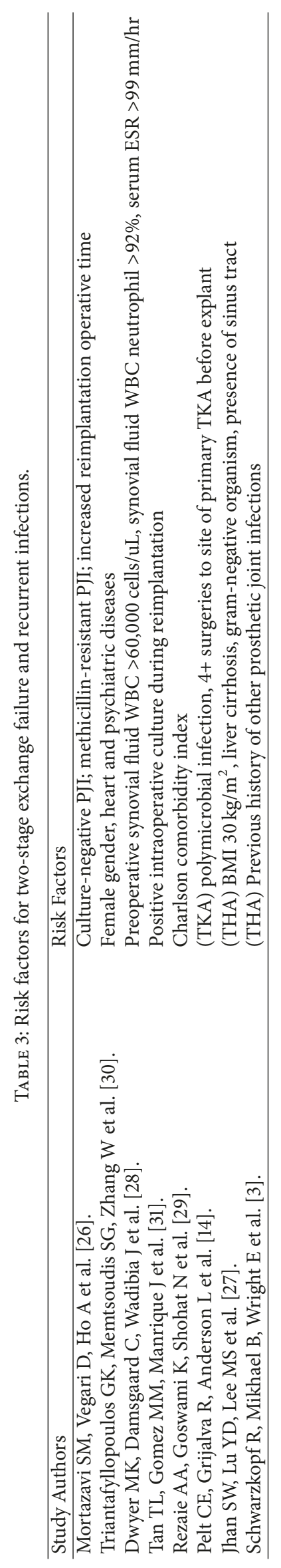


TABLE 4: Prognostic factors for predicting sepsis mortality after two-stage exchange.

Study Authors

Prognostic Factors

Srisangthong P, Wongsa A, Kittiworawitkul P and

Wattanathum A [32].

Plasma IL-6 100 pg/mL (28-day mortality)

Parlato M, Cavaillon JM [33].

Lactate, pentraxin 3 (PTX3), pancreatic stone protein, IL-8; microRNA markers 15a/16/193/483-5p, CD14 and CD64 (28-day mortality)

Rodelo JR, De la Rosa G, Valencia ML et al. [34]. D-dimer (28-day mortality)

Artero A, Zaragoza R, Zamarena JJ et al. [35]. Hypoalbuminemia

Chen Y, Chunsheng L [36].

tic peptide $>113 \mathrm{pg} / \mathrm{mL}$ (28-day mortality)

Ranzani OT, Zampieri FG, Forte DN et al. [37]; Kim

$\mathrm{MH}$, Ahn JY, Song JE et al. [38].

Plasma brain natriuretic peptide $>113 \mathrm{pg} / \mathrm{mL}$ (28-day mortality)

CAR (90-day [37] and 180-day [38] mortality)

ratio, which encompasses the inflammatory and nutritional status of a patient, is not a significant indicator of the outcomes of two-stage exchanges after PJI. This is in contrast to the value of CAR as shown in cancer patients.

There were several limitations to this retrospective study. First, the number of patients that met the inclusion criteria was small despite using two different institutional databases. The lack of significant difference of CAR between the successful and failed two-stage exchange was possibly limited by this factor. Secondly, lab values collected up to a year prior to a patient's explant were used to analyze CAR which could not accurately represent the patient's physical state prior to receiving surgery. Finally, patients who had revisions prior to their explant for their two-stage exchange were included, which could also negatively impact the outcomes.

Despite these pitfalls, this study contributes to the literature by applying a successful prognostic model into a field that needs more tools to improve its outcomes. It evaluated the outcomes of two-stage exchanges for THA and TKA in patients from two different institutions who were diagnosed with PJI per the MSIS definition using a novel preoperative systemic-inflammatory prognostic score, CAR. Based on our knowledge of the inflammatory process in PJI and in tumor progression, we hypothesized a correlation, but no significant difference was found with the numbers available. It also highlights the need to prospectively study CAR or other preoperative systemic-inflammation prognostic scores in the setting of PJI as it may provide value in helping to identify the correct timing of reimplantation.

\section{Conclusion}

This study aims to analyze the predictability of two-stage exchange outcomes to treat PJI defined by the MSIS criteria using a novel preoperative systemic-inflammatory score, CAR. Patient data was collected from two institutional databases and CAR did not predict success or failure after a two-stage exchange in the setting of a PJI. Despite the limitations and conclusion, this study provides insight and explores the prognostic possibilities of preoperative inflammatory scores in the setting of PJIs as they have been previously shown to be successful in other surgical field managing diseases driven by inflammation. Further research is needed to explore the application of other scores, such as neutrophil/lymphocyte, in order to better predict a patient's outcome after a two-stage exchange and to alleviate the financial and emotional burden of PJI.

\section{Data Availability}

The patient data used to support the findings of this study are restricted by the local Institutional Review Board in order to protect patient privacy.

\section{Disclosure}

An earlier version of this work has been presented at the MSIS Annual Meeting 2018.

\section{Conflicts of Interest}

The authors declare that there are no conflicts of interest regarding the publication of this paper.

\section{References}

[1] S. M. Kurtz, K. L. Ong, E. Lau, and K. J. Bozic, "Impact of the economic downturn on total joint replacement demand in the United States," The Journal of Bone and Joint Surgery, vol. 96, no. 8, pp. 624-630, 2014.

[2] S. M. Kurtz, E. Lau, H. Watson, J. K. Schmier, and J. Parvizi, "Economic burden of periprosthetic joint infection in the United States," The Journal of Arthroplasty, vol. 27, no. 8, supplement 1, pp. 61.e1-65.el, 2012.

[3] R. Schwarzkopf, B. Mikhael, E. Wright, D. M. Estok II, and J. N. Katz, "Treatment failure among infected periprosthetic total hip arthroplasty patients," The Open Orthopaedics Journal , vol. 8, no. 1, pp. 118-124, 2014.

[4] A. J. Tande and R. Patel, "Prosthetic joint infection," Clinical Microbiology Reviews, vol. 27, no. 2, pp. 302-345, 2014.

[5] H. Sharma, J. De Leeuw, and D. I. Rowley, "Girdlestone resection arthroplasty following failed surgical procedures," International Orthopaedics, vol. 29, no. 2, pp. 92-95, 2005.

[6] V. Khanna, D. M. Tushinski, L. J. Soever, A. D. Vincent, and D. J. Backstein, "Above knee amputation following total knee arthroplasty: when enough is enough," The Journal of Arthroplasty, vol. 30, no. 4, pp. 658-662, 2015.

[7] M. Wouthuyzen-Bakker, J. M. Nijman, G. A. Kampinga, S. van Assen, and P. C. Jutte, "Efficacy of antibiotic suppressive therapy 
in patients with a prosthetic joint infection," Journal of Bone and Joint Infection, vol. 2, no. 2, pp. 77-83, 2017.

[8] H. J. Cooper and C. J. Della Valle, "The two-stage standard in revision total hip replacement," The Bone \& Joint Journal, vol. 95-B, no. 11_Supple_A, pp. 84-87, 2013.

[9] T. Mahmud, M. C. Lyons, D. D. Naudie, S. J. MacDonald, and R. W. McCalden, "Assessing the gold standard: a review of 253 twostage revisions for infected TKA," Clinical Orthopaedics and Related Research, vol. 470, no. 10, pp. 2730-2736, 2012.

[10] J. Petrie, A. Sassoon, and G. Haidukewych, "Two-stage revision for the infected total knee arthroplasty: the gold standard," Seminars in Arthroplasty, vol. 24, no. 3, pp. 149-151, 2013.

[11] A. N. Ford, A. M. Holzmeister, H. W. Rees, and P. D. Belich, "Characterization of outcomes of 2-stage exchange arthroplasty in the treatment of prosthetic joint infections," The Journal of Arthroplasty, vol. 33, no. 7, pp. S224-S227, 2018.

[12] F. Y. Sabry, L. Buller, S. Ahmed, A. K. Klika, and W. K. Barsoum, "Preoperative prediction of failure following two-stage revision for knee prosthetic joint infections," The Journal of Arthroplasty, vol. 29, no. 1, pp. 115-121, 2014.

[13] S. Bozhkova, R. Tikhilov, D. Labutin et al., "Failure of the first step of two-stage revision due to polymicrobial prosthetic joint infection of the hip," Journal of Orthopaedics and Traumatology, vol. 17, no. 4, pp. 369-376, 2016.

[14] C. E. Pelt, R. Grijalva, L. Anderson et al., "Two-stage revision TKA is associated with high complication and failure rates," Advances in Orthopedics, vol. 2014, Article ID 659047, 7 pages, 2014.

[15] M. Shibutani, K. Maeda, H. Nagahara et al., "Prognostic significance of the preoperative ratio of c-reactive protein to albumin in patients with colorectal cancer," Anticancer Research, vol. 36, pp. 995-1001, 2016.

[16] M. M. Elahi, D. C. McMillan, C. S. McArdle, W. J. Angerson, and N. Sattar, "Score based on hypoalbuminemia and elevated c-reactive protein predicts survival in patients with advanced gastrointestinal cancer," Nutrition and Cancer, vol. 48, no. 2, pp. 171-173, 2004.

[17] Y. Oshiro, R. Sasaki, K. Fukunaga et al., "Inflammation-based prognostic score is a useful predictor of postoperative outcome in patients with extrahepatic cholangiocarcinoma," Journal of Hepato-Biliary-Pancreatic Sciences, vol. 20, no. 3, pp. 389-395, 2013.

[18] Y. Liang, W. Xiao, Y. Guan et al., "Prognostic value of the C-reactive protein/Albumin Ratio (CAR) in patients with operable soft tissue sarcoma," OncoTargets and Therapy, vol. 10, pp. 98135-98147, 2017.

[19] X. Xu, H. Yu, W. Hu, Q. Song, and W. Mao, "A novel inflammation-based prognostic score, the C-reactive protein/albumin ratio predicts the prognosis of patients with operable esophageal squamous cell carcinoma," PLoS ONE, vol. 10, no. 9, 2015.

[20] M. Ishizuka, H. Nagata, K. Takagi, Y. Iwasaki, N. Shibuya, and K. Kubota, "Clinical significance of the C-reactive protein to albumin ratio for survival after surgery for colorectal cancer," Annals of Surgical Oncology, vol. 23, pp. 900-907, 2015.

[21] K. Haruki, H. Shiba, Y. Shirai et al., "The C-reactive protein to albumin ratio predicts long-term outcomes in patients with pancreatic cancer after pancreatic resection," World Journal of Surgery, vol. 40, pp. 2254-2260, 2016.

[22] J. Parvizi and T. Gehrke, "Definition of periprosthetic joint infection," The Journal of Arthroplasty, vol. 29, no. 7, p. 1331, 2014.
[23] C. Diaz-Ledezma, C. A. Higuera, and J. Parvizi, "Success after treatment of periprosthetic joint infection: a delphi-based international multidisciplinary consensus," Clinical Orthopaedics and Related Research, vol. 471, no. 7, pp. 2374-2382, 2013.

[24] B. Zmistowski, J. A. Karam, J. B. Durinka, D. S. Casper, and J. Parvizi, "Periprosthetic joint infection increases the risk of oneyear mortality," The Journal of Bone and Joint Surgery-American Volume, vol. 95, no. 24, pp. 2177-2184, 2013.

[25] Y. S. Lee and A. F. Chen, "Two-stage reimplantation in infected total knee arthroplasty," Knee Surgery and Related Research, vol. 30, no. 2, pp. 107-114, 2018.

[26] S. M. J. Mortazavi, D. Vegari, A. Ho, B. Zmistowski, and J. Parvizi, "Two-stage exchange arthroplasty for infected total knee arthroplasty: predictors of failure," Clinical Orthopaedics and Related Research, vol. 469, no. 11, pp. 3049-3054, 2011.

[27] S. Jhan, Y. Lu, M. S. Lee, C. Lee, J. Wang, and F. Kuo, “The risk factors of failed reimplantation arthroplasty for periprosthetic hip infection," BMC Musculoskeletal Disorders, vol. 18, no. 1, 2017.

[28] M. K. Dwyer, C. Damsgaard, J. Wadibia et al., "Laboratory tests for diagnosis of chronic periprosthetic joint infection can help predict outcomes of two-stage exchange," The Journal of Bone and Joint Surgery, vol. 100, no. 12, pp. 1009-1015, 2018.

[29] A. Aali Rezaie, K. Goswami, N. Shohat, A. T. Tokarski, A. E. White, and J. Parvizi, "Time to reimplantation: waiting longer confers no added benefit," The Journal of Arthroplasty, vol. 33, no. 6, pp. 1850-1854, 2018.

[30] G. K. Triantafyllopoulos, S. G. Memtsoudis, W. Zhang, Y. Ma, T. P. Sculco, and L. A. Poultsides, "Periprosthetic infection recurrence after 2-stage exchange arthroplasty: failure or fate?" The Journal of Arthroplasty, vol. 32, no. 2, pp. 526-531, 2017.

[31] T. L. Tan, M. M. Gomez, J. Manrique, J. Parvizi, and A. F. Chen, "Positive culture during reimplantation increases the risk of subsequent failure in two-stage exchange arthroplasty," The Journal of Bone and Joint Surgery, vol. 98, no. 15, pp. 1313-1319, 2016.

[32] P. Srisangthong, A. Wongsa, P. Kittiworawitkul, and A. Wattanathum, "Early IL-6 response in sepsis is correlated with mortality and severity score," Critical Care, vol. 17, no. Suppl 2, p. P34, 2013.

[33] M. Parlato and J. Cavaillon, "Host response biomarkers in the diagnosis of sepsis: a general overview," Journal of Molecular Biology and Methods, vol. 1237, pp. 149-211, 2015.

[34] J. R. Rodelo, G. De la Rosa, M. L. Valencia et al., "D-dimer is a significant prognostic factor in patients with suspected infection and sepsis," The American Journal of Emergency Medicine, vol. 30, no. 9, pp. 1991-1999, 2012.

[35] A. Artero, R. Zaragoza, J. J. Camarena, S. Sancho, R. González, and J. M. Nogueira, "Prognostic factors of mortality in patients with community-acquired bloodstream infection with severe sepsis and septic shock," Journal of Critical Care, vol. 25, no. 2, pp. 276-281, 2010.

[36] Y. Chen and C. Li, "Prognostic significance of brain natriuretic peptide obtained in the ED in patients with SIRS or sepsis," The American Journal of Emergency Medicine, vol. 27, no. 6, pp. 701706, 2009.

[37] O. T. Ranzani, F. G. Zampieri, D. N. Forte, L. C. P. Azevedo, and M. Park, "C-reactive protein/albumin ratio predicts 90-day mortality of septic patients," PLoS ONE, vol. 8, no. 3, Article ID e59321, 2013.

[38] M. H. Kim, J. Y. Ahn, J. E. Song et al., "The C-reactive protein/albumin ratio as an independent predictor of mortality 
in patients with severe sepsis or septic shock treated with early goal-directed therapy," PLoS ONE, vol. 10, no. 7, Article ID e0132109, 2015.

[39] L. Fagotti, J. Tatka, M. J. Salles, and M. C. Queiroz, "Risk factors and treatment options for failure of a two-stage exchange," Current Reviews in Musculoskeletal Medicine, vol. 11, no. 3, pp. 420-427, 2018.

[40] D. C. Sing, T. F. Luan, B. T. Feeley, and A. L. Zhang, "Is obesity a risk factor for adverse events after knee arthroscopy?" Arthroscopy: The Journal of Arthroscopic and Related Surgery, vol. 32, no. 7, pp. 1346-1353.el, 2016.

[41] K. L. Hackman, M. J. Bailey, G. I. Snell, and L. A. Bach, "Diabetes is a major risk factor for mortality after lung transplantation," American Journal of Transplantation, vol. 14, no. 2, pp. 438-445, 2014.

[42] Y. Liu, X. He, J. Pan, S. Chen, and L. Wang, "Prognostic role of Glasgow prognostic score in patients with colorectal cancer: evidence from population studies," Scientific Reports, vol. 7, no. 1, 2017.

[43] Y. Yu, L. Qian, and J. Cui, "Value of neutrophil-to-lymphocyte ratio for predicting lung cancer prognosis: a meta-analysis of 7,219 patients," Molecular and Clinical Oncology, vol. 7, no. 3, pp. 498-506, 2017.

[44] C. I. Diakos, K. A. Charles, D. C. McMillan, and S. J. Clarke, "Cancer-related inflammation and treatment effectiveness," The Lancet Oncology, vol. 15, no. 11, pp. e493-e503, 2014.

[45] D. C. Angus and T. van der Poll, "Severe sepsis and septic shock," The New England Journal of Medicine, vol. 369, no. 9, pp. 840851, 2013.

[46] X. Liu, X. Sun, J. Liu et al., "Preoperative C-reactive protein/albumin ratio predicts prognosis of patients after curative resection for gastric cancer," Translational Oncology, vol. 8, no. 4, pp. 339-345, 2015.

[47] M. S. Cha, S. H. Cho, D. H. Kim et al., "Two-stage total knee arthroplasty for prosthetic joint infection," Knee Surgery and Related Research, vol. 27, no. 2, pp. 82-89, 2015. 


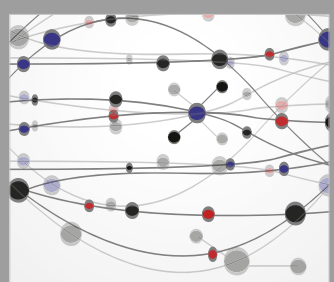

The Scientific World Journal
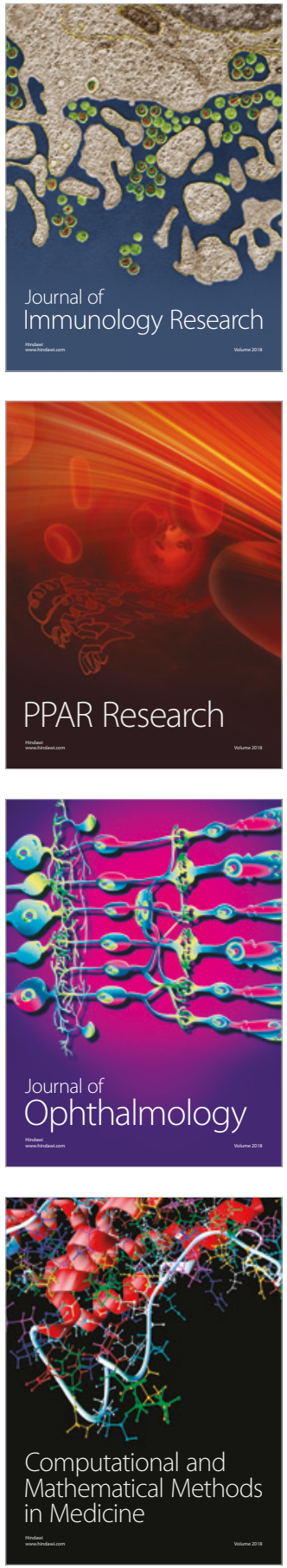

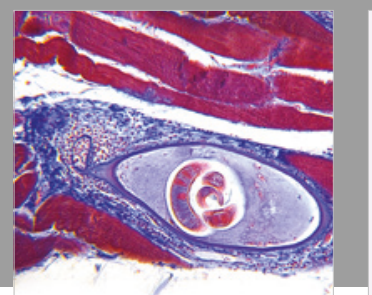

Gastroenterology Research and Practice

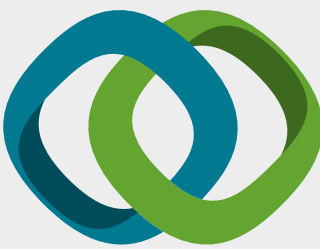

\section{Hindawi}

Submit your manuscripts at

www.hindawi.com
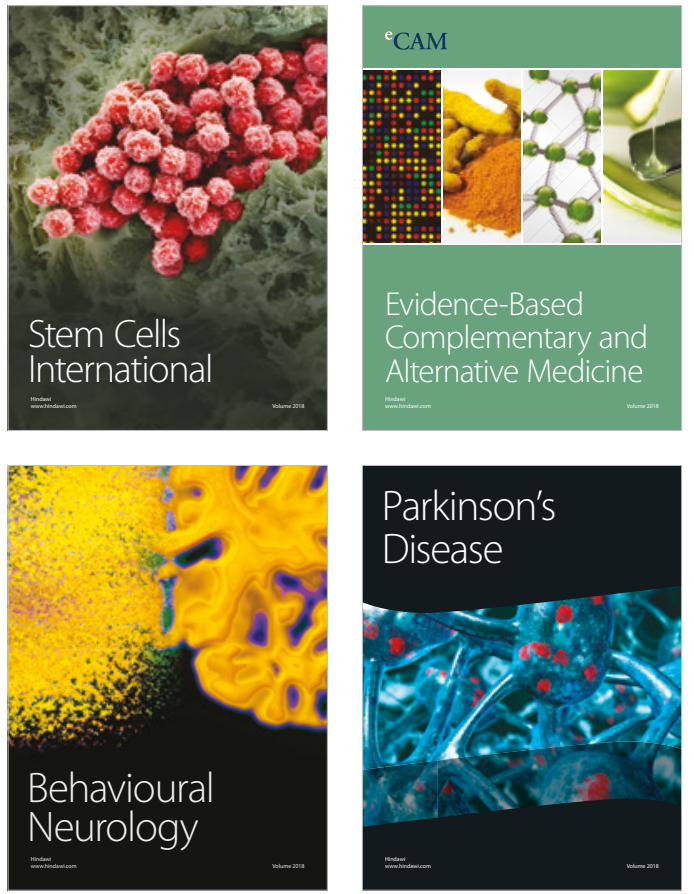

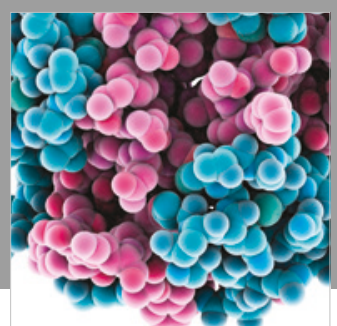

ournal of

Diabetes Research

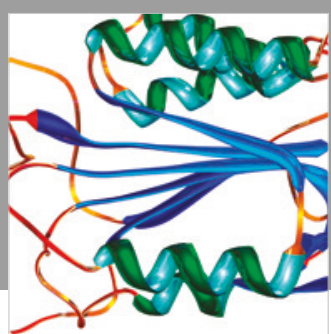

Disease Markers
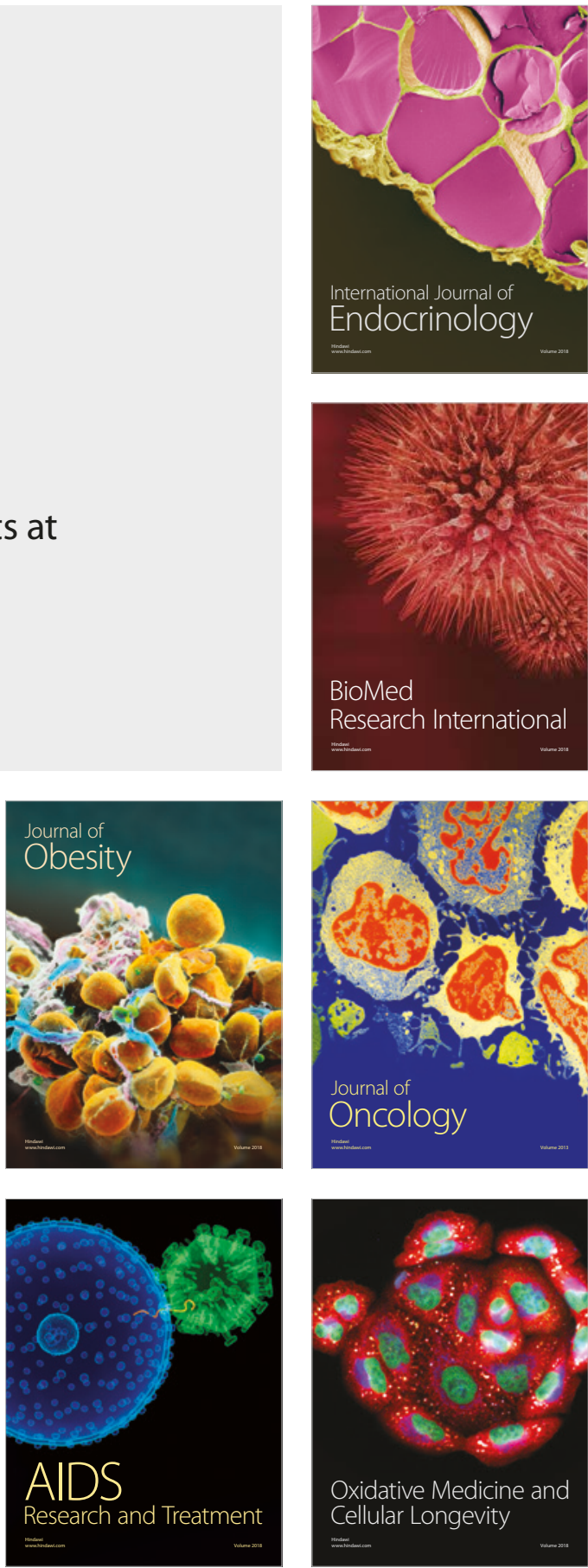